\title{
Networks as integrated in research methodologies in PER
}

\author{
Jesper Bruun \\ Department of Science Education, University of Copenhagen, Oester Voldgade 3, Copenhagen, Denmark
}

\begin{abstract}
In recent years a number of researchers within the PER community have started using network analysis as a new methodology to extend our understanding of teaching and learning physics by viewing these as complex systems. In this paper, I give examples of social, cognitive, and action mapping networks and how they can be analyzed. In so doing I show how a network can be methodologically described as a set of relations between a set of entities, and how a network can be characterized and analyzed as a mathematical object. Then, as an illustrative example, I discuss a relatively new example of using networks to create insightful maps of learning discussions. To conclude, I argue that conceptual blending is a powerful framework for constructing "mixed methods" methodologies that may integrate diverse theories and other methodologies with network methodologies.
\end{abstract}

\section{INTRODUCTION}

This text is based on a presentation given at PERC2016 [1]. It is an introduction to network theory as a methodological tool in physics education research. I wish to stress here that the scope of the paper is to give examples of how to use networks in PER. I do not wish to give a comprehensive review of network analysis as it has been used in physics education research. Nor is the scope of this paper a detailed analysis of how networks affect research methodologies. Network analysis in PER is still a very new endeavor and we have yet to see the impact of this methodological tool. I write this paper in the hope that researchers in PER will consider the potentially very broad range of application that I believe network analysis to have.

\section{BOUNDARY CONDITIONS FOR NETWORKS AS A RESEARCH METHODOLOGY}

A network is a collection of entities and a set of corresponding connections. In network terminology [2], the entities are called nodes or vertices depending on the field. The connections are either called links or edges and directed links and arcs if the direction of connections is relevant/determinable. See Fig. 1.

From a methodological perspective, working with networks imposes a set of boundary conditions. First, the particular phenomenon, system, or object under investigation will be projected onto a network. Any such projection is a reduction, which emphasizes the relational structure inherent to the phenomenon, system, or object; the projection will split the phenomenon, system, or object into parts, and it re-emerges as a network. Second, networks have a history; they were created in some way, and they may change when influenced by some kind of event. The networks we investigate are a product of that history, which means that we can expect networks to be very different, even if they are meant to describe the same phenomenon. Third, and lastly, networks are heterogeneous, meaning that each node is a unique entity. It is unique because of how it is connected to other nodes in the

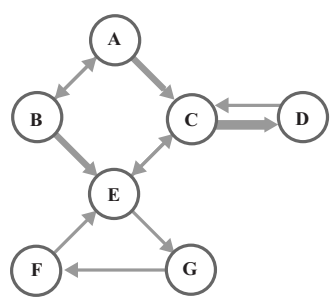

(A)

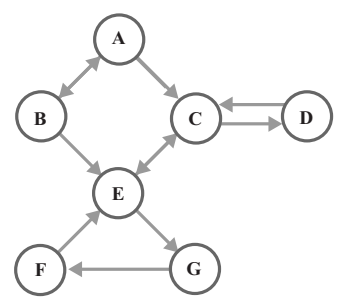

(C)

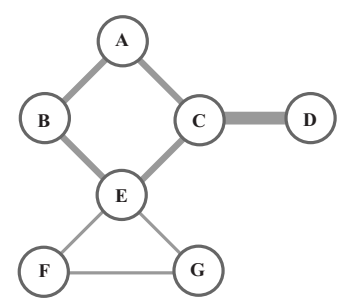

(B)

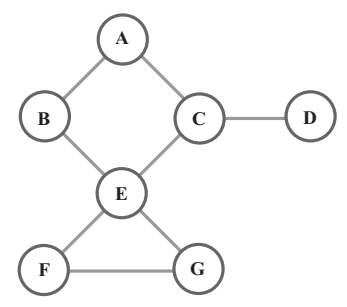

(D)

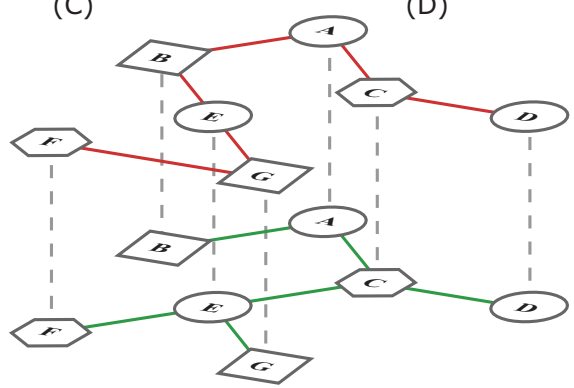

(E)

FIG. 1. Different kinds of networks. (A) A weighted and directed network. (B) A weighted and undirected network. (C) A unweighted and directed network. (D) An unweighted and undirected network. (E) A multiplex or layered network. Each layer contains nodes of different kinds.

network. A network methodology can take this uniqueness into account, which in turn may provide a very detailed analysis of the phenomenon, system, or object. The following section describes examples of how network methodologies can shape the theoretical insights that can be gained from different types of implementation. These insights depend on the 
particular choices that researchers make for what represents a node, what represents a link, and how structures might be interpreted in terms of an underlying theoretical basis. The examples will demonstrate how network methodologies bring out and emphasize the relational aspects of what has been studied.

\section{EXAMPLES OF NETWORKS IN RESEARCH METHODOLOGIES}

In this section, I provide three contemporary examples of how Network Analysis may be employed as a methodological tool. The emphasis is on the kinds of choices a researcher will make with regards to analysis and interpretation when working with networks.

\section{A. Social networks}

This example draws attention to the visual and the mathematical aspects of networks. The setting for this example is a Danish upper secondary physics class. For seven weeks during the fall period, students were prompted to indicate with whom they communicated about physics. Each week would give a different network with a directed link from student $\mathrm{A}$ to student B, if A had chosen B on the roster. Network diagrams of two of these networks can be seen in Fig. 2(A,B). The prompt had been developed using student reports on their collaboration and a Communities of Practice (CoP) [4] informed framework (see [3] for details).

To utilize the power of social network analysis, it is often a good idea to bring in information that is external to the network. The nodes that represent boys in Fig. 2 are purple and the nodes that represent girls are green. Links between girls are green, links between boys are purple, and inter-gender links are black.

The visual side of the network diagrams clearly shows that boys and girls are separate with few inter-gender links and the boys forming a community of their own. Furthermore girls seem to interact much more during the test week (B) as compared with the lab week (A).

The node sizes are proportional to the target entropy, which is a measure of the un-predictability of information coming to a node in a network. Large target entropy means less predictability. Target entropy has previously been related to academic success [5]. This measure of centrality can be interpreted both on a node level basis and-because entropy is additive- on a whole-network level. This is done by comparing the whole-network target entropy for the network in question with a large number of randomized versions of the network (see [3] and references therein for details). By doing so for different weeks one can see patterns in the data. For example, it seems that every time this class engages with laboratory experiments, the target entropy drops (as compared

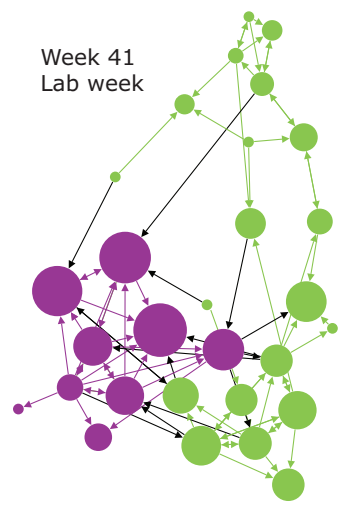

(A)

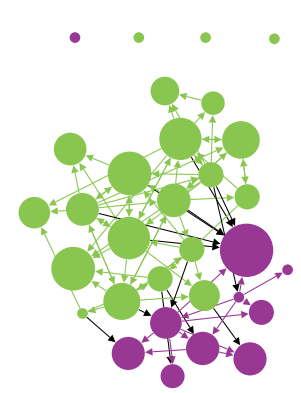

Week 45 Test week

(B)
Target entropy variation

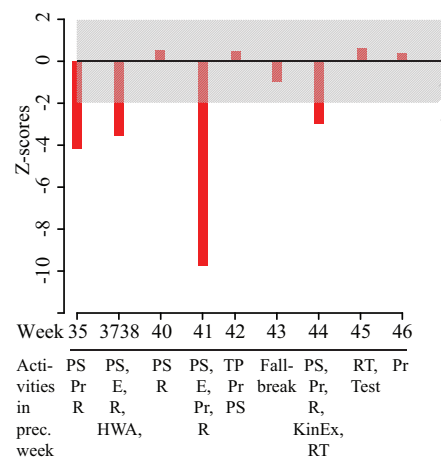

(C)

FIG. 2. A and B show two network diagrams of student interactions from two different weeks from the same classroom. The number of nodes and links in each network are roughly the same. Green circles represent girls, purple circles represent boys. Black links represent interactions across gender. Node size is proportional to target entropy. C shows the Z-score of target entropy for different weeks in relation to the teaching-learning activities in class. See [3].

with randomized versions), meaning that it is easier to predict from where a student gets information.

These results can be used to create candidate rules of interaction [6]. Here, one candidate rule of interaction could be: "When doing experimental work, these students are unlikely to share their experiences outside a small group." From a Communities of Practice (CoP) perspective, the class as a whole may not fruitfully be treated as a CoP. Rather, smaller groups may be CoPs. This insight was driven by the mathematical nature of these networks.

\section{B. Cognitive networks}

This example shows how Network Analysis provides for a new way of looking at questionnaire data. From a methodological view point, I wish to show two things. First, because of the relational structure of networks, we can also ask questions that link to theories, which hypothesize a relational 


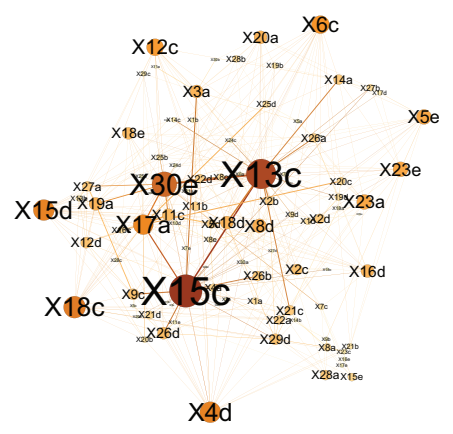

(A)

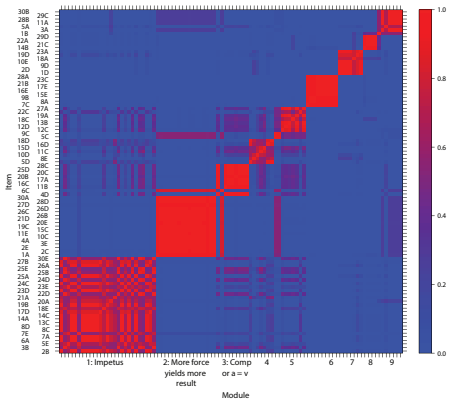

(B)

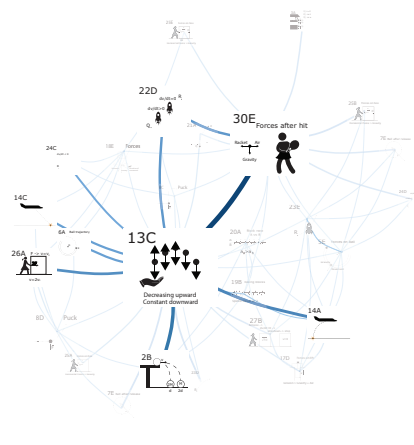

(C)

FIG. 3. (A) A network of FCI response items based on student answers. (B) A matrix describing how often two items are grouped together in a module. (C) The impetus module. Sub-figures have been adapted from Brewe et al. [7] under the creative commons license (https: //creativecommons.org/licenses/by/3.0/legalcode).

structure. Second, networks may or may not be viewed as systems in which information can flow and this distinction leads to different kinds of questions.

Based on student post responses $(N \approx 150)$ to the Force Concept Inventory (FCI, [8]), we constructed a network of incorrect response items [7]. Two items are connected if at least one student has indicated both items. Figure 3 A shows a backbone of this network, meaning only the strongest connections as seen from the individual node perspective. There is a strong connection (thick line) between $13 \mathrm{C}$ and $30 \mathrm{E}$, indicating that students who chose $13 \mathrm{C}$ also tended to choose $30 \mathrm{E}$.

To find patterns in the data, one can employ a community detection algorithm, Infomap [9], to partition the network into modules that are more densely connected to each other than to the rest of the network. Infomap can be described as a random walker traversing the network by following links. If the walker is 'trapped' for a period of time in a particular part of the network, that part becomes a module. One of these modules is shown in Fig. $3 \mathrm{C}$. This has been labeled an 'impetus cluster', because many of the items resemble what has been called an impetus understanding of Newtonian mechanics [7]. However, we also discover that many of the response items are only weakly connected to this cluster. A first indication of this stems from the fact that many of the connections (lines) are thin. This is important because Infomap works by optimizing a quality function, and this may result in slightly different partitions each time Infomap is run. Running Infomap 1000 times, it is apparent the impetus cluster is not clearly defined (the bottom square in the diagonal of Fig. 3 $\mathrm{C}$ is not clearly red, but has many purple bits); it has a welldefined core (red portions in the bottom square in Fig. 3 B), but many of the items are often grouped into other modules. This contrasts the second module (the next square on the diagonal), which is well-defined.

From a theory perspective, one could link this finding to, for example, the conceptual change discussion of knowledge in pieces versus knowledge as theory [10]. A knowledge as pieces perspective might be linked to a fuzzy cluster such as the impetus-cluster in Fig. $3 \mathrm{~B}$ and $\mathrm{C}$, whereas a coherent theory might be linked to clusters that are well-defined and form a coherent whole. This would require a deep analysis of both the results and of student reasons for making the choices they made.

From a methodological perspective, this network as compared with the networks in Fig. $2 \mathrm{~A}+\mathrm{B}$, contrasts between viewing networks as structural entities or as entities connected via information flow. The assumption for the target entropy measure is the latter, and it seems appropriate for student interaction networks, because one can imagine that information flowing (although not unchanged) when students communicate. In the response item network (Fig. $3 \mathrm{~A}$ ), information might be seen as flowing in the sense that one response led to another. A contrasting view could be that nodes that are linked act together, so that the groups found represent networked cognitive structures. I believe that these two different ways of viewing this network would lead to different kinds of questions asked. In the first view, one might ask what makes a person choose particular items when having chosen particular other items. In the second view, one might ask what characterizes the cognitive structure.

\section{C. 'Action' mapping networks}

The purpose of this example is to show how data that describes some kind of behavior can be treated as a network. This example pertains to how students click on a web page, but the general idea can be extended to more complex behaviors, such as students interacting each other in a learning situation [12] or how they answer questions during an interview [13]. With this example, I wish to highlight that Network Analysis of behaviors offers a novel way of analyzing the structure of the occurrence of behaviors.

In this example, students interacted with problems in an 


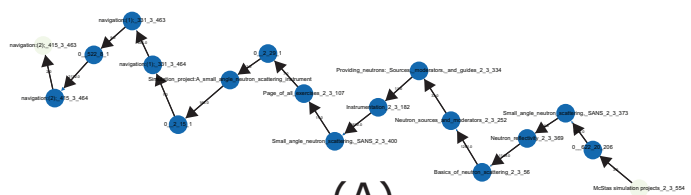

(A)

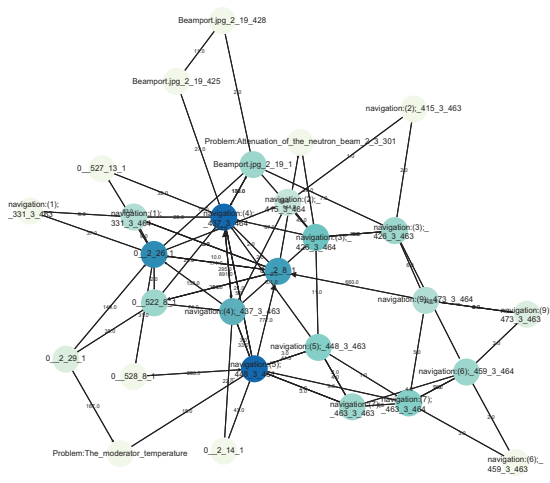

(B)

FIG. 4. (A) A network with zero target entropy. (B) A network with high target entropy. From [11].

online textbook by navigating via mouse clicks. For example, they could show hints and solutions to problems by clicking buttons. If they subsequently wanted to hide hints and solutions from view, they would click on hide hint/solution [11]. Students would primarily solve these problems during class either alone or in small groups. Other navigational clicks on the web-page were also recorded, meaning that it could be seen how they navigated the entire online textbook.

Here, clicks on links on the page were used as the basis for creating networks; clickable text or graphics were represented as nodes and links on the order of clicks. Two nodes are connected in the network if one link was clicked after the other. We defined sessions by using server log data, and we converted sessions to networks based on recorded clicks. Figure $4 \mathrm{~B}$ and $\mathrm{C}$ show two different types of session networks.

In this study, we made the theoretically informed choice that clicks on show/hide hint/solution were to be recorded as particular nodes. By being able to track students' way of using hints and solutions we might infer aspects of how these kinds of problems can act as an artifact that students can use to internalize problem solving skills in a Vygotskian sense [14].

From a methodological perspective, an important point is that other types of behavior could be mapped, such as behaviors discernible from video, audio or classroom observations. All of these actions can in principle be mapped by order of occurrence or by dividing the data into chunks and then mapping co-occurrence $[12,13,15]$.

\section{A RELATIVELY NEW EXAMPLE: MAPPING DISCUSSIONS}

The following example shows a novel way of integrating network analysis with qualitative discourse analysis to develop a bottom-up systematic approach to generating themes in data. The case is a student discussion of sustainability, which illustrates how this integration can make the analysis more sensitive to different voices in the discussion.

\section{A. The cycle}

Imagine a transcript of a group discussion. All references to individual students have been removed, so the transcript only contains information about what was said in the space of the discussion. Lindahl et. al [16] proposes a cyclic method for integrating network analysis with qualitative discourse analysis to produce a thematic map of the discussion. The cycle starts with conducting a qualitative discourse analysis and by creating a linguistic network [17] of the discussion where a directed link is drawn from word $\alpha$ to word $\beta$ if $\alpha$ precedes $\beta$ in the transcript (Fig. 5 A1). Then Infomap [9] is used to partition the linguistic network into a map of connected modules (Fig. 5 A2). This is a candidate thematic map, and the modules are candidate themes to be interpreted in terms of the results from the discourse analysis. We call this process a trial characterization. This may result in changes in the discourse analysis. However, it is likely that the network analysis and the discourse analysis cannot be aligned in a meaningful manner. In that case, the trial characterization will serve as the basis for constructing rules for changes in the transcript. These rules can be removal of common words, merging of synonyms or grammatical categories, and concatenating words to phrases with specific meaning. Once the rules have been made they are applied throughout the transcript, resulting in a new linguistic network (Fig. 5 A2) and a new candidate map with new candidate modules (Fig. 5 B2). At some point the thematic map and the qualitative discourse analysis should converge, and the result is a final thematic map with an associated interpretation (Fig. 5 C). See [16] and [18] for details.

\section{B. Example: Sensitivity to different voices}

As part of a teaching sequence, a group of Swedish students were to discuss conflicting views presented in a newspaper article about the inbreeding of wolves in Sweden. Initially for this group, the qualitative discourse analysis revealed a discussion that appeared mostly one-sided. It seemed dominated by a single point-of-view (protect the Swedish wolves by using fences). The one-sideness in the discussion apparently left little room for additional exploration.

This picture changed when we iteratively integrated network analysis; the thematic constructs the discussion is 


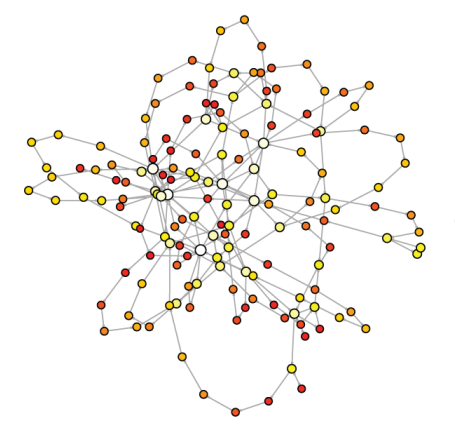

154 words

220 links

(A1)

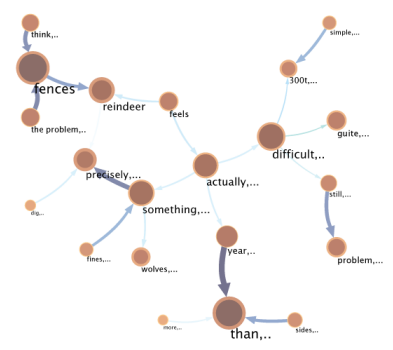

(A2)

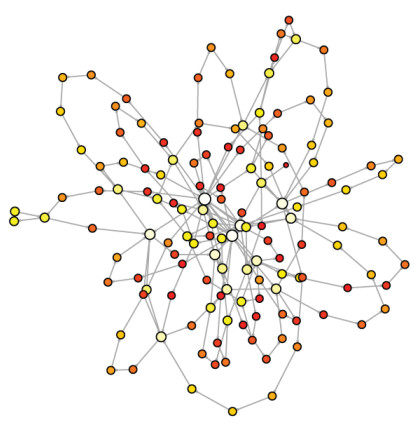

139 words

(B1)

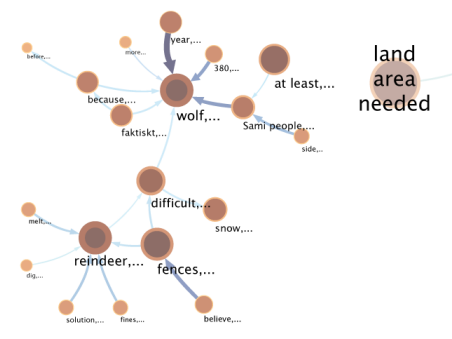

(B2)

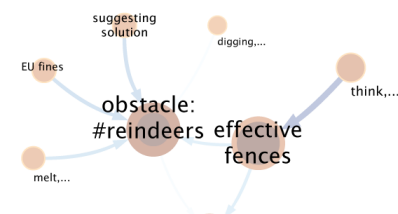

snow difficulties

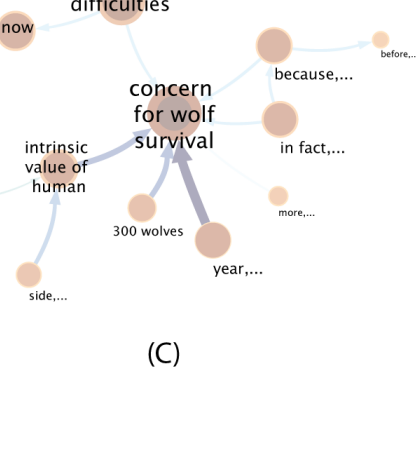

(1)

FIG. 5. (A1) A network from one of the cycles aligning discourse with network analysis. (A2) The corresponding trial thematic map. (B1) The final network. (B2) The final thematic map. (C) The final thematic map with interpretation. From [16].

weaved around are: concern for wolf survival,\#reindeers, effective fences and difficulties. See Fig. 5C. The final map shows two distinct subsystems (there are no strong connections between the two sets of modules in Fig. 5C) indicating that the structure of the discussion was not one-sided. The one centered on concern for wolf survival, and the other, $o b$ stacle: \#reindeers, being centered on obstacles arising from issues relating to effective fences and the numbers of reindeers. So instead of a one-sided discussion, this analysis revealed more of a tug-of-war between obstacles, being more concrete, and intrinsic values, being more abstract. For further illustrations and details, see Bruun, Lindahl, and Linder (2016) [18].

\section{INTEGRATING NETWORK ANALYSIS IN MIXED RESEARCH METHODOLOGIES}

Throughout this text, I have attempted to draw attention to some of the choices researchers have to make when working with networks. These are quite fundamental and will have consequences for the interpretations and explanations that network methodologies will provide for. While any detailed reflections about these choices are beyond the scope of this paper, Table I summarizes these choices.

\section{A. Blend spaces and mixed methodologies}

Working with networks in physics education research has very much to do with finding out what can be represented and how that affects the study. I have found the idea of conceptual blending $[19,20]$ helpful when construing methodologies involving networks. Since methodology is intimately linked to theory, one can start with linking concepts from educational theory to the concepts from network science. For example, students, knowledge pieces, parts of self-efficacy, or types of teaching/learning activities might be linked to nodes, while student relationships or similarity, triggering of concepts, and sequences of teaching/learning activities might be mapped to links. What is important here is that not everything from the domain of network analysis and not everything from the domains of educational theory gets to be mapped in any single study. And furthermore, we can expect that the new domain, whatever we want to call it, will have new entities of interest emerge [19]. For example, the idea of 'rules of interaction' in the sense that it has been discussed in recent PER literature $[3,6]$ is new. In this literature, 'rules of interaction' are social norms from which observable network structures will likely emerge. Furthermore, the new domain contains a merger between educational theory and the mathematical and visual parts of networks (illustrated in Fig. 6). And the same kind of reasoning holds true for other methodologies. 
TABLE I. Things to keep in mind when working with networks.

\begin{tabular}{|c|c|}
\hline $\begin{array}{l}\text { Thing to keep } \\
\text { in mind }\end{array}$ & Explanation \\
\hline $\begin{array}{l}\text { What nodes and } \\
\text { links represent }\end{array}$ & $\begin{array}{l}\text { This is an ongoing negotiation and should } \\
\text { be treated dynamically throughout the } \\
\text { research process. }\end{array}$ \\
\hline $\begin{array}{l}\text { Networks as flows or } \\
\text { scaffolding structures }\end{array}$ & $\begin{array}{l}\text { These two different views will facilitate } \\
\text { different kinds of questions that researchers } \\
\text { ask the data. }\end{array}$ \\
\hline $\begin{array}{l}\text { Networks as } \\
\text { mathematical objects }\end{array}$ & $\begin{array}{l}\text { Network theory offers a myriad of ways } \\
\text { of doing calculations on micro-, meso- and } \\
\text { macroscopic levels. }\end{array}$ \\
\hline $\begin{array}{l}\text { Modeling dynamics } \\
\text { on networks }\end{array}$ & $\begin{array}{l}\text { This line of research would investigate e.g. } \\
\text { how information spreads in a network } \\
\text { - taking the network nodes and links as given }\end{array}$ \\
\hline $\begin{array}{l}\text { Modeling dynamics } \\
\text { of networks }\end{array}$ & $\begin{array}{l}\text { This line of research would investigate how } \\
\text { networks form and evolve in terms of nodes } \\
\text { and links between them. }\end{array}$ \\
\hline $\begin{array}{l}\text { Network science is } \\
\text { evolving rapidly }\end{array}$ & $\begin{array}{l}\text { New developments occur almost every day } \\
\text { in many areas of network science. }\end{array}$ \\
\hline $\begin{array}{l}\text { Analysis] } \\
\text { Codes } \\
\text { Dependencies } \\
\text { Themes }\end{array}$ & $\begin{array}{c}\text { Education } \\
\text { Theory } \\
\text { Concepts } \\
\text { Relationships } \\
\text { Hypothesized } \\
\text { Structures }\end{array}$ \\
\hline
\end{tabular}

FIG. 6. A simplified version of conceptual blending network science with educational theory and other research methodologies.

From the perspective of conceptual blending, researchers who embrace network methodologies will likely use their existing knowledge in conjunction with knowledge of network science to interpret both visual and mathematical results, to construct new ways of visualizing network calculations, and to construct mathematical models of learning situations informed by networks.

\section{B. Final remarks}

Network Analysis is new territory in physics education research. Many methodological questions involving Network Analysis as methodology are unanswered and will need to be investigated. For example, what are the relationships between Network Analysis and statistical methods such as factor analysis, clustering, and item response theory? Does Network Analysis qualify as a methodology (or as an integral part of methodology) as I have argued or is it merely a new form of representation? As a methodological lens, what does Network Analysis allow researchers to capture, and what is left overlooked by these kinds of analyses? And perhaps most importantly, how can Network Analysis contribute to the development of theories and design of education to improve people's learning of physics? Hopefully, in the years to come, many researchers will help provide answers to these questions.

\section{ACKNOWLEDGMENTS}

In this paper, I have mostly referenced work that I have been involved with. This is solely because I find it easier to explain my own work than the work of others. Therefore, I want to acknowledge the work of Ismo T. Koponen and his group at the University of Helsinki, the work of Madelin Bodin at the University of Umeå, the work of Cedric Linder and Jonas Forsman at the University of Uppsala, and many others. I have included references at the end of this paper that might guide the reader to other relevant network studies.

\section{Appendix: Additional references}

The following references appeared in the talk and are not otherwise included in the reference section of this paper.

- Virginia Braun and Victoria Clarke, "Using thematic analysis in psychology," Qualitative Research in Psychology 3, 77-101 (2006).

- Eric Brewe, Laird Kramer, and Vashti Sawtelle, "Investigating student communities with network analysis of interactions in a physics learning center," Physical Review Special Topics-Physics Education Research 8, 010101 (2012).

- Jesper Bruun, Networks in physics education research: A theoretical, methodological and didactical explorative study, Ph.D. thesis, University of Copenhagen (2012).

- Jesper Bruun and Ian Bearden, "Time development in the early history of social networks: Link stabilization, group dynamics, and segregation," PLOS One 9, e112775 (2014).

- Alan J. Daly, ed., Social Network Theory and Educational Change (Harvard Education Press, Cambridge, MA, 2010). 
- Ane von der Fehr, Sølberg, and Jesper Bruun, "Validation of networks derived from snowball sampling of municipal science education actors," International Journal of Research \& Method in Education , 1-15 (2016).

- Jonas Forsman, Rachel Moll, and Cedric Linder, "Extending the theoretical framing for physics education research: An illustrative application of complexity science," Physical Review Special Topics-Physics Education Research 10, 020122 (2014).

- Renee Michelle Goertzen, Eric Brewe, and Laird Kramer, "Expanded markers of success in introductory university physics," International Journal of Science Education 35, 262-288 (2013).

- Jesper Juul Jensen, Formativ evaluering $i$ Almen Studieforberedelse, Master's thesis, Department of Sci- ence Education, University of Copenhagen (2015).

- Ismo T. Koponen and Tommi Kokkonen, "A systemic view of the learning and differentiation of scientific concepts: The case of electric current and voltage revisited," Frontline Learning Research 2, 140-166 (2014).

- Ismo T. Koponen, Tommi Kokkonen, and Maija Nousiainen, "Dynamic systems view of learning a threetiered theory in physics: Robust learning outcomes as attractors," Complexity (2016).

- Maija Nousiainen and Ismo T. Koponen, "Concept maps representing knowledge of physics: Connecting structure and content in the context of electricity and magnetism," Nordic Studies in Science Education 6, 155-172 (2010).
[1] Jesper Bruun, "Network analysis as a research methodology in PER," (2016), invited talk at 2016 Physics Education Research Conference.

[2] Mark Newman, Networks: An Introduction (Oxford University Press, Oxford; New York, 2010).

[3] Jesper Bruun, Networks in physics education research: A theoretical, methodological and didactical explorative study, Ph.D. thesis, University of Copenhagen (2012).

[4] Etienne Wenger, Communities of Practice: Learning, Meaning, and Identity (Cambridge University Press, 1998).

[5] Jesper Bruun and Eric Brewe, "Talking and learning physics: Predicting future grades from network measures and Force Concept Inventory pretest scores," Physical Review Special Topics-Physics Education Research 9, 020109 (2013).

[6] Jonas Forsman, Cedric Linder, Rachel Moll, Duncan Fraser, and Staffan Andersson, "A new approach to modelling student retention through an application of complexity thinking," Studies in Higher Education 39, 68-86 (2014).

[7] Eric Brewe, Jesper Bruun, and Ian G. Bearden, "Using module analysis for multiple choice responses: A new method applied to Force Concept Inventory data," Physical Review Physics Education Research 12, 020131 (2016).

[8] David Hestenes, Malcolm Wells, and Gregg Swackhamer, "Force Concept Inventory," The Physics Teacher 30, 141-158 (1992).

[9] Martin Rosvall and Carl T. Bergstrom, "Maps of random walks on complex networks reveal community structure," Proceedings of the National Academy of Sciences 105, 1118-1123 (2008).

[10] Gökhan Özdemir and Douglas B. Clark, "An overview of conceptual change theories," Eurasia Journal of Mathematics, Science \& Technology Education 3, 351-361 (2007).

[11] Jesper Bruun, P. Jensen, and Linda Udby, "Mapping student online actions," (2015), poster presented at Complenet 2015, New York, United States.

[12] Julie Hougaard, Using virtual experiments as a preparation for large scale facility experiments., Master's thesis, Department of Science Education, University of Copenhagen (2015).

[13] Madelen Bodin, "Mapping university students' epistemic framing of computational physics using network analysis," Physical Review Special Topics-Physics Education Research 8, 010115 (2012).

[14] Lev S. Vygotsky, Mind in Society: The Development of Higher Mental Process (Harvard University Press, Cambridge, MA, 1978).

[15] David Williamson Shaffer, David Hatfield, Gina Navoa Svarovsky, Padraig Nash, Aran Nulty, Elizabeth Bagley, Ken Frank, André A Rupp, and Robert Mislevy, "Epistemic network analysis: A prototype for 21st-century assessment of learning," International Journal of Learning and Media 1, 3353 (2009).

[16] Mats Lindahl, Jesper Bruun, and Cedric Linder, "Maps of student discussions about sustainability," (2016), invited poster presentation at 2016 Physics Education Research Conference.

[17] A. P. Masucci and G. J. Rodgers, "Network properties of written human language," Physical Review E 74, 026102 (2006).

[18] Mats Lindahl, Jesper Bruun, and Cedric Linder, "Forthcoming," (2016).

[19] Gilles Fauconnier and Mark Turner, "Conceptual blending, form and meaning," Recherches en Communication 19, 57-86 (2003).

[20] Noah S. Podolefsky and Noah D. Finkelstein, "Analogical scaffolding and the learning of abstract ideas in physics: An example from electromagnetic waves," Physical Review Special Topics-Physics Education Research 3, 010109 (2007). 